\title{
Underwater endoscopic submucosal dissection in saline solution with rubber-band countertraction for a cecal polyp extending into a diverticulum
}

\author{
Georgios Mavrogenis ${ }^{a}$, loannis Mavrogenis ${ }^{a}$, Stylianos Anastasiadis ${ }^{a}$, Fateh Bazerbachi ${ }^{b}$ \\ Mediterraneo Hospital, Athens, Greece; Mayo Clinic, Rochester, USA
}

A 50-year-old female patient was referred for resection of a 4 -cm serrated polyp of the cecum (Paris IIa) with partial extension into a diverticulum (Fig. 1). After submucosal injection, the part of the lesion that extended into the diverticulum was lifted and partially dissected (PCF 190L, DualKnifeJ, Olympus, Tokyo, Japan) (Fig. 2). A clip with a rubber band (3/16", RMO Inc, Denver, USA) was then applied at the intradiverticular edge of the lesion and the circumferential incision was completed. The rubber band was grasped with a second clip and was tethered to the opposite colonic wall. In this fashion, constant traction was applied and the submucosal space was readily exposed. In addition, the lumen was filled with normal saline to improve the lifting of the lesion as well as the endoscopic view. At the end of the procedure, the specimen was detached from the rubber with a loop cutter (Olympus) and the mucosal defect, including the diverticular orifice, was closed with clips. The patient had an uneventful recovery. Histology showed R0 resection of a serrated adenoma with low-grade dysplasia.

Three previous reports of colon endoscopic submucosal dissection (ESD) in the setting of diverticular involvement described the clip and line technique [1], the pocket creation method [2,3], and the inversion of the diverticulum with a cap [2]. However, these techniques are either complex or not always feasible. Based on our experience, the combination of rubber-band traction with underwater ESD could be an alternative option for safer dissection of polyps with intradiverticular extension.

\section{Acknowledgment}

The authors would like to thank Dr Mathieu Pioche for demonstrating the rubber-band traction technique in ESGE

Department of a Gastroenterology, Mediterraneo Hospital, Athens, Greece; bastroenterology and Hepatology, Mayo Clinic, Rochester, USA

Conflict of Interest: None

Correspondence to: Georgios Mavrogenis, Kavetsou 10, 81100

Mytilene, Greece, e-mail: mavrogenis@gmail.com

Received 1 May 2019; accepted 12 May 2019;

published online 20 June 2019

DOI: https://doi.org/10.20524/aog.2019.0396

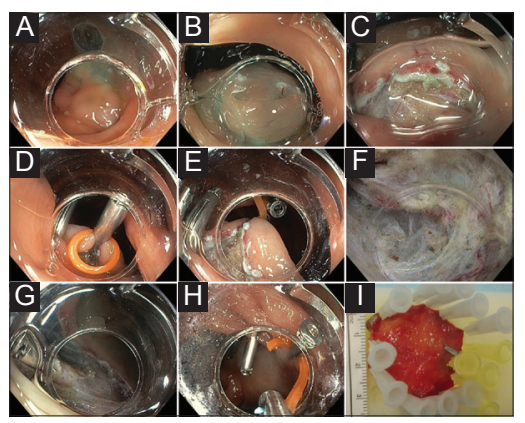

Figure 1 Intraprocedural images. (A) The anal site of the polyp extends into a diverticulum. (B) Lifting of the intradiverticular part of the lesion. (C) Creation of a flap. (D) Placement of a clip with rubber band over the flap. (E) Attachment of the flap at the opposite side of the colon. (F) Underwater dissection. (G) Mucosal defect. (H) Cutting the rubber band. (I) Resected specimen

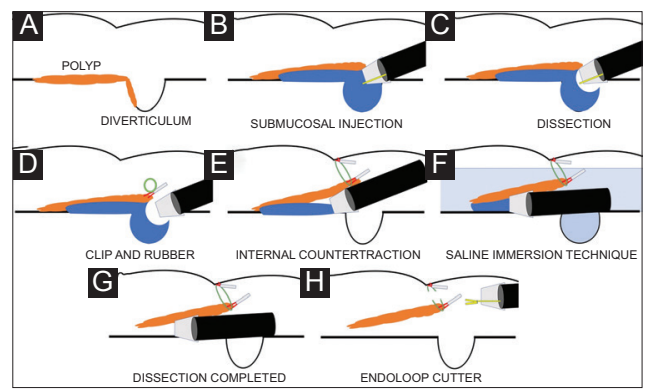

Figure 2 Schematic representation of the strategy. (A) Polyp with partial extension into a diverticulum. (B) Elevation of the polyp. (C) Detachment of the polyp from the diverticulum. (D) Application of a clip with a rubber band at the intradiverticular edge of the polyp.

(E) Fixation of the flap at the colonic wall. (F) Underwater dissection.

(G) Completion of the resection. (H) Cutting the rubber band

days 2019, as well as Dr Edwart Despott for sharing his experience with underwater ESD in the social media.

\section{References}

1. Shichijo S, Yamasaki Y, Takeuchi Y. Case of colonic adenoma involving a diverticulum resected by a traction-assisted endoscopic submucosal dissection technique. Dig Endosc 2017;29:729-730.

2. Yoshida N, Naito Y, Kishimoto M. Endoscopic submucosal dissection of T1 cancer with colonic diverticulum by pocketcreation method. Dig Endosc 2017;29:726-727.

3. Arimoto J, Chiba H, Tachikawa J, Goto T, Nakajima A. New inversion method: endoscopic submucosal dissection of a neoplasm extending into a colonic diverticulum. VideoGIE 2019;4:230-231. 